\title{
Application of the PODFS method to inlet turbulence generated using the digital filter technique
}

\author{
N. C. W. Treleaven ${ }^{\mathrm{a}, \mathrm{b}, *}$, M. Stauferc ${ }^{\mathrm{c}}$ A. Spencer ${ }^{\mathrm{b}}$, A. Garmory ${ }^{\mathrm{b}}$, G. J. Page ${ }^{\mathrm{b}}$ \\ ${ }^{a}$ Simulation of reactive Thermo-Fluid Systems, TU-Darmstadt, 64287 Darmstadt, \\ Germany \\ ${ }^{b}$ Department of Aeronautical and Automotive Engineering, Loughborough University, \\ Loughborough, LE11 3TU, United Kingdom \\ ${ }^{c}$ Rolls-Royce Deutschland Ltd \& Co KG, 15827 Blankenfelde-Mahlow, Germany
}

\section{Abstract}

In the past the digital filter technique has been used to successfully generate inflow turbulence for a number of academic and industrially relevant reacting and non-reacting flows. Weaknesses of the method include the requirement that the filter be computed over a structured mesh and can require extremely long computation times in cases where the turbulent length scales or filter widths are large as compared to the mesh spacing. Once computed, the inflow data may be saved and reused, however tens-of-thousands of time steps worth of data must be saved, copied and re-loaded into memory for each new computation and temporal interpolation must be used if the time step is adjusted. The newly developed PODFS (proper orthogonal decomposition Fourier series) method uses proper orthogonal decomposition (POD) to compress this large data set into a handful of modes that are optimally chosen to represent the high energy turbulent structures while a Fourier series rep-

\footnotetext{
${ }^{*}$ Corresponding author

Email address: treleaven@stfs.tu-darmstadt.de (N. C. W. Treleaven )
} 
resentation of the temporal components of the POD modes means that the data becomes continuous, periodic and flexible in terms of time step. The PODFS is first applied to a set of inflow data generated using the digital filter method and used to simulate a turbulent planar jet with the results compared against a DNS simulation. A practical application of the method is then demonstrated where it is used to generate inlet turbulence from a time averaged URANS profile for a swirl stabilised combustor case. In this case, two PODFS models are linearly combined, one that represents acoustic forcing from downstream and one that represents turbulent fluctuations. This highlights a feature of the method which is its ability to represent different flow phenomena using the linear addition of two or more PODFS models. A subsequent LES calculation shows that the method results in the correct penetration of the airflow jets whilst neglecting the inlet turbulence results in the incorrect jet penetration depth.

Keywords:

LES, DNS, turbulence, digital filters, inlet boundary condition

\section{Introduction}

In order to reduce the cost of numerical simulations of turbulent flows the size of the computational domain is often reduced to include only the region of interest. As the physical size of the simulation reduces, the results obtained become increasingly sensitive to the boundary conditions, and this is particularly important for unsteady flows. Examples of such situations include simulations of jet engine combustors where the system inlet flow is disturbed by the upstream turbomachinery which may be simulated by a 
single stage and allowance must be made for the effects of upstream stages or cooling flows [1, 2, 3, 4].

Several different methods have been developed in order to provide suitable upstream boundary conditions for scale resolving simulations such as LES (Large Eddy Simulation) and DNS (Direct Numerical Simulation). These methods can be broadly divided into three types: Recycling methods, synthetic turbulence methods and volume forcing methods [5]. Another interesting application of these methods is in the field of hybrid LES-RANS (Reynolds Averaged Navier-Stokes) simulations where turbulence must be created out of the RANS statistical quantities such as the turbulent kinetic energy and the rate of energy dissipation [3, 5].

Recycling methods use an auxiliary simulation to produce the required turbulent field which is then copied and scaled to be applied to the main simulation. This includes the strong recycling method where the turbulent fields generated in an auxillary scale resolved simulation are copied directly to the inlet of the main simulation [6, 7, 8]. Alternatively the main and auxiliary simulations can be combined and run together as in the weak recycling method. In this case, turbulent fields downstream in the domain are extracted, re-scaled and re-applied at the inlet [9, 10, 3]. The inlet data can be re-used in a subsequent simulation, but running a high resolution DNS simulation of a channel flow for the purpose of providing an inlet boundary condition for a reacting LES simulation is not computationally efficient. Alternative methods aim to produce the turbulence as it is required.

These so called synthetic inflow turbulence methods generate pseudoturbulence that contains the correct statistical and spectral information with- 
out resorting to solving the Navier-Stokes simulations directly. One of the most popular of these methods is the digital filter technique first pioneered by Bechara et al. [11] and further developed by Klein et al. [12]. The method generates random uncorrelated fluctuations which are then filtered to obtain the correct spatial and temporal correlations. The method has been shown to provide representative inlet turbulent fields for planar jets [13], simulations of primary breakup of a liquid jet [12], combusting LES [14], shock wave and boundary layer interaction [15] and CAA simulations [16] among others. Other synthetic inflow turbulence methods include the synthetic random Fourier method [17, 11, 18, 19] and the synthetic coherent eddy method [20, 21, 22, 23].

A third category of turbulence generators is the volume forcing type. These methods generate turbulence, not by applying velocity fluctuations at the inlet, but by imposing additional body forces in the initial region of the flow to promote the development of turbulence. An example is the work of Spille-Kohoff and Kaltenbach [24] where a wall-normal body force was added in a control region near the inlet of the domain for the simulation of a turbulent boundary layer. The magnitude of the body force for each cell was chosen to follow a prescribed turbulent energy spectrum. The integral magnitude of these fluctuations was chosen using feedback control that aimed to obtain the desired Reynolds shear stress profile at a downstream location. Similarly to the weak recycling method, a disadvantage of these methods is the need to include an upstream region where the flow turbulence can develop before reaching the critical region of interest, increasing computational cost. A more comprehensive discussion on the various different kinds of turbulence 
generators is provided in [5].

There are several practical drawbacks to the digital filter method: Whilst cheaper than a DNS, the computational cost of the method can be high. For a $50 \times 400$ inlet mesh and a filter length of 16 mesh points, each inlet required over a minute to be computed on the single core of an Intel Core i5 processor. LES or DNS simulations can require hundreds of thousands of time steps and as such the time required for the computation of the inlet turbulent field is significant. Many simulations are similar which means that the same inlet data may be stored and used in subsequent simulations, however, if the simulation timestep is altered then the data must be re-sampled at the correct frequency. For the same $50 \times 400$ inlet mesh, each inlet field (written in single precision ASCII format) required two megabytes of storage space. This means that for a full simulation potentially gigabytes of inlet data must be stored and copied for each new simulation. The time required for loading of these files at runtime is also significant, especially in the case of massively parallel simulations where many processors may have to load the same data simultaneously. The digital filter method is most easily computed over a structured regular mesh which means that the fields must be interpolated onto the unstructured mesh (typically required for industrial geometries) further increasing errors and computational cost. These restrictions may be eased through the use of more intelligent file types, better code integration and memory sharing, however, such efforts can also be applied to the method described here which further decreases the computational cost of the digital filter method.

The unsteady PODFS method computes a low order semi-empirical, semi- 
analytical representation of a set of inlet data and has been shown to correctly reproduce the effect of acoustic forcing on jet engine combustion chambers [25]. The method computes the POD (Proper Orthogonal Decomposition) of the original data which results in a collection of modes that are optimal in the sense that it is the decomposition that reproduces the maximum kinetic energy with the minimal number of modes. Only a relatively small number of these modes are required to capture the large scale energy containing turbulent structures reducing the amount of data that must be stored, copied and loaded by several orders of magnitudes. The POD modes are composed of a temporal and spatial component (or spatial modes and temporal coefficients). While the temporal coefficients are independent of space, the spatial components are independent of time and may be re-used at each time step meaning that the loading of the spatially varying information and interpolation onto the unstructured mesh has only to be computed once at the beginning of the simulation. In order to represent the temporal components, a Fourier series is chosen as this ensures that the method is continuous, periodic and may be used to reproduce the original data using any size of time step. Whist the PODFS method is used in this case to compress turbulent fields generated by the digital filter method, it can also be used to compress any applied turbulent inlet field such as one that is generated using the strong recycling method.

The POD has previously been used to reproduce turbulent inlets using linear stochastic estimation (LSE) [26] and a low-order dynamical system (LODS) approach [27]. The advantage of the PODFS method over the LSE approach is that the LSE approach requires an input signal to generate future 
snapshots of the turbulent inlet, and as such cannot be used to represent future inlet states. The LODS approach relies on a low-order dynamical system to generate future inlet states. The dynamical system is based on the incompressible Navier-Stokes equations which are unlikely to accurately represent the dynamics of non-turbulent phenomena such as the effect that acoustic forcing has on the inlet field. The use of a Fourier series to represent the POD temporal coefficients is more general, more stable and can be applied to any signal providing that it can be assumed to be temporally pseudo-periodic.

This paper aims to show how combining the digital filter method with PODFS significantly improves the usefulness of the technique for industrially relevant scale resolving simulations such as LES. It begins with a description of the digital filter methodology in Section 2 and then the PODFS method in Section 3. This includes a description about how multiple PODFS models can be combined to represent turbulent and periodic inlet fluctuations. This is especially useful in simulations where multiple unsteady sources must be considered in order to represent an accurate boundary condition. One example of this situation is at the inlet of a combustion chamber where acoustic waves present in the flow may cause periodic variations to the inlet velocity while upstream components will generate turbulence. Another example might be at the inlet of a turbine blade row where the upstream boundary condition includes the turbulent field contribution and periodic variations due to the upstream nozzle guide vanes. There are three test cases: in Section 4, simple DNS channel flow data is reproduced using the digital filter and PODFS to show how the data compression affects the accuracy of the inflow statistics. In Section 5, a highly resolved simulation of a planar jet at 
a Reynolds number of $R e=4000$ is run with the turbulent inlet flow generated using the digital filter PODFS technique and compared to the same case run with the original data produced with the digital filter method. These results are then compared to the DNS results of Stanley et al. [28] with references made to the original digital filter simulations run by Klein et al. [13, 12. Finally in Section 6, an LES simulation of an acoustically forced gas turbine fuel injector is run to highlight the ability of the PODFS method to reproduce both the periodic effects of forcing and the PODFS digital filter method to produce sufficient inflow turbulence to correct the penetration of the central injector jet flow. The motivation for such a test is that the digital filter PODFS method enables the use of incompressible simulations to be used instead of much more expensive compressible simulations to examine flame dynamics in gas turbine combustion systems. The paper also includes an appendix that describes how to get access to and use the digital_filters.py python program to produce the PODFS models used in this paper.

\section{The digital filter method}

For completeness, and as a starting point for the PODFS, the digital filter method as described by Klein et al. [12] is outlined here. There are three steps to the digital filter method: The first is the generation of random data, then the data is filtered to impose the correct length scales and then finally the magnitude of the fluctuations are adjusted to the required Reynolds stress tensor. 


\subsection{Generation of the random field}

A three dimensional random field $(r)$ is generated for each velocity component with dimensions $2 \times N_{x}+1,2 \times N_{y}+1+J, 2 \times N_{z}+1+K$ where $N_{x}, N_{y}, N_{z}$ are the half filter support lengths in the inlet normal direction, and the two in-plane directions respectively. $J$ and $K$ are the number of grid points in the in-plane directions. The $x$-direction corresponds to the temporal coordinate. At each time step the first $y, z$-plane is discarded and all of the data shifted from $i, j, k$ to $i-1, j, k$. A new $N_{y}+1+J, 2 \times N_{z}+1+K$ random field is generated and added to the $N_{x}, j, k$ index of $r$ where $-N_{x}<i<N_{x}$. The random field must have the properties that $\overline{r_{i}}=0$ and $\overline{r_{i} r_{j}}=\delta_{i j}$ where $r_{i}$ is the $i$ th field realisation and the over-bar represents the average over all $i$.

\subsection{Filtering}

In order to impose the correct length scales onto this uncorrelated random field, a three dimensional digital filter is applied at each grid point:

$$
\tilde{\mathbf{u}}_{m, n}=\sum_{i=-N_{x}}^{N_{x}} \sum_{j=-N_{y}}^{N_{y}} \sum_{k=-N_{z}}^{N_{z}} b_{i, j, k} \mathbf{r}_{i, m+j, n+k} \text { for } 1<m<J \text { and } 1<n<K
$$

The three dimensional filter coefficients $\left(b_{i, j, k}\right)$ are built from three, one dimensional filters which assume that the autocorrelation of velocity takes the form [29]:

$$
R_{u u}(r, 0,0)=\exp \left(-\frac{\pi}{4} \frac{r^{2}}{L_{x}^{2}}\right)
$$

where $L_{x}$ is the integral length scale in the x-direction. The corresponding filter coefficients for this correlation are given by: 


$$
b_{i} \approx \tilde{b}_{i} /\left(\sum_{p=-N_{x}}^{N_{x}} \tilde{b}_{p}^{2}\right)^{1 / 2}
$$

where:

$$
\tilde{b}_{i}=\exp \left(-\frac{\pi}{2} \frac{i^{2}}{N_{x}^{2}}\right)
$$

The filter width should be at least twice the length scale. The three dimensional filter coefficients are then found using:

$$
b_{i, j, k}=b_{i} \times b_{j} \times b_{k}
$$

The length scales in the principle directions that lie in the inlet plane should be chosen so they are representative of the length scales found in a similar flow as observed in experiment or DNS. At each timestep, a new random field is generated and the filter reapplied. The time step between each newly generated turbulent field and the inlet resolution are linked to the plane normal mean velocity using Taylor's hypothesis:

$$
\Delta t_{f}=\frac{\Delta x}{\overline{u_{x}}}
$$

In cases where the simulation time step $(\Delta t)$ is different to the one implied by the above relation, the resulting snapshots produced by the digital filter method can be resampled at the required frequency using an interpolation strategy. The other option is to adjust the inlet normal direction length scale such that: 


$$
L_{x}=\frac{\Delta x}{\overline{u_{x}} \Delta t} L_{x 0}
$$

where $L_{x 0}$ is the original desired length scale. This strategy has the advantage that less fields must be generated and the length scale and therefore filter widths are smaller. This is obviously limited by the requirement that there is spatial and temporal correlation between each temporal snapshot. In the limit of the filter width becoming equal to 1 , the correlation between each random field drops to zero.

\subsection{Adaption to the Reynolds stress profile}

Different inlets will also have different Renolds stress tensors. In order to take this into account the approach of Lund et al. [10] is used: The filtered velocity fluctuations are transformed using $u_{i}=\bar{U}_{i}+a_{i j} \tilde{u}_{j}$ with

$$
\left[a_{i j}\right]=\left[\begin{array}{ccc}
R_{11}^{1 / 2} & 0 & 0 \\
R_{21} / a_{11} & \left(R_{22}-a_{21}^{2}\right)^{1 / 2} & 0 \\
R_{33} / a_{11} & \left(R_{32}-a_{21} a_{31}\right) / a_{22} & \left(R_{33}-a_{31}^{2}-a_{32}^{2}\right)^{1 / 2}
\end{array}\right]
$$

where $R_{i j}$ is the required Reynolds stress tensor. Different donor simulations or experiments will produce approximations of the Reynolds stress tensor. In the case of scale resolved simulations or Reynolds stress model URANS or RANS simulations, the full Reynolds stress tensor may be available to be use with the digital filter method, in many cases, only part of the tensor will be resolved. In these cases, the Reynolds stress tensor may be approximated by using the Boussinesq eddy viscosity approach: 


$$
R_{i j}=-\nu_{t}\left(\frac{\partial U_{i}}{\partial x_{j}}+\frac{\partial U_{j}}{\partial x_{i}}\right)+\frac{2}{3} k \delta_{i j}
$$

where $\nu_{t}$ is the turbulent viscosity. In the case of turbulent closure using $k-\omega$ type models it can be approximated using $\nu_{t}=k / \omega$.

\section{The PODFS method}

In order to produce a low order PODFS model of the turbulent field, the snapshot proper orthogonal decomposition (POD) method [30] is applied to the fluctuating velocity components of the inlet plane coming from the digital filter method:

$$
\mathbf{u}\left(\mathbf{x}, t_{k}\right)=\sum_{i=1}^{N_{s}} a_{i}\left(t_{k}\right) \phi_{i}(\mathbf{x})
$$

where $t_{k}$ is the $k$ th timestep, $a_{i}\left(t_{k}\right)$ are the POD temporal coefficients, $\phi_{i}(\mathbf{x})$ are the POD spatial modes and $N_{s}$ is the number of snapshots. The PODFS reconstruction of the data from the output of the digital filter is given by:

$$
\begin{array}{r}
\tilde{U}_{i n}\left(\mathbf{x}, t, E_{\lambda}, E_{b}\right)=\alpha \bar{U}_{i n}+ \\
\sum_{i=0}^{N_{P}} \phi_{i}\left(\mathbf{x}_{i n}\right) \sum_{j=0}^{N_{F_{i}}-1} b_{i l(j)} e^{2 \pi \imath l(j) t / \Delta t N_{S}}
\end{array}
$$

where $N_{F_{i}}$ is the number of Fourier mode coefficients used for the $i$ th POD mode, and $N_{p}$ is the number of POD modes used in the reconstruction. $\alpha$ is a small correction factor that can be used to account for errors in the inter-

polation of the data. In the case of PODFS models built using idealised inlet 
conditions, $\alpha$ should be set to 1 , however in cases where the PODFS model is generated using fluctuating inlet data from simulation or experiment, $\alpha$ can be used to correct the mean flow velocity or mean mass flow rate back to the value obtained in the original simulation.

In order to produce a low order model of the data, the temporal coefficients are approximated using a Fourier series:

$$
\tilde{a}_{i}(t) \approx \sum_{j=0}^{N_{F_{i}}-1} b_{i l(j)} e^{2 \pi \imath l(j) t / \Delta t N_{S}}
$$

where $l(j)$ is a ranking function that ranks the Fourier modes according to the magnitude of their coefficients. The Fourier coefficients $\left(b_{i j}\right)$ are calculated using:

$$
\begin{aligned}
& b_{i j}=\frac{1}{N_{S}} \sum_{k=0}^{N_{S}-1} a_{i}\left(t_{k}\right) e^{-2 \pi \imath j t_{k} / \Delta t N_{S}}, \\
& j=-N_{S} / 2, \ldots, N_{S} / 2-1
\end{aligned}
$$

The number of POD modes used to reproduce the original data $\left(N_{P}\right)$ are governed by the desired POD reconstruction energy:

$$
E_{\lambda}\left(N_{P}\right)=\frac{\sum_{i=1}^{N_{P}} \lambda_{i}}{\sum_{i=1}^{N_{S}} \lambda_{i}}
$$

where $\lambda_{i}$ is the eigenvalue of the $i$ th POD mode and the number of Fourier coefficients $\left(N_{F_{i}}\right)$ is defined by the required Fourier mode energy:

$$
E_{b}\left(N_{F_{i}}, i\right)=\frac{\sum_{j=0}^{N_{F_{i}}}\left|b_{i l(j)}\right|}{\sum_{j=0}^{N_{S}-1}\left|b_{i l(j)}\right|}
$$


The PODFS method has been shown to be much more computationally efficient than the strong recycling method in [25] and has the advantage that as the temporal modes are represented as periodic analytical functions, any timestep or running time may be used.

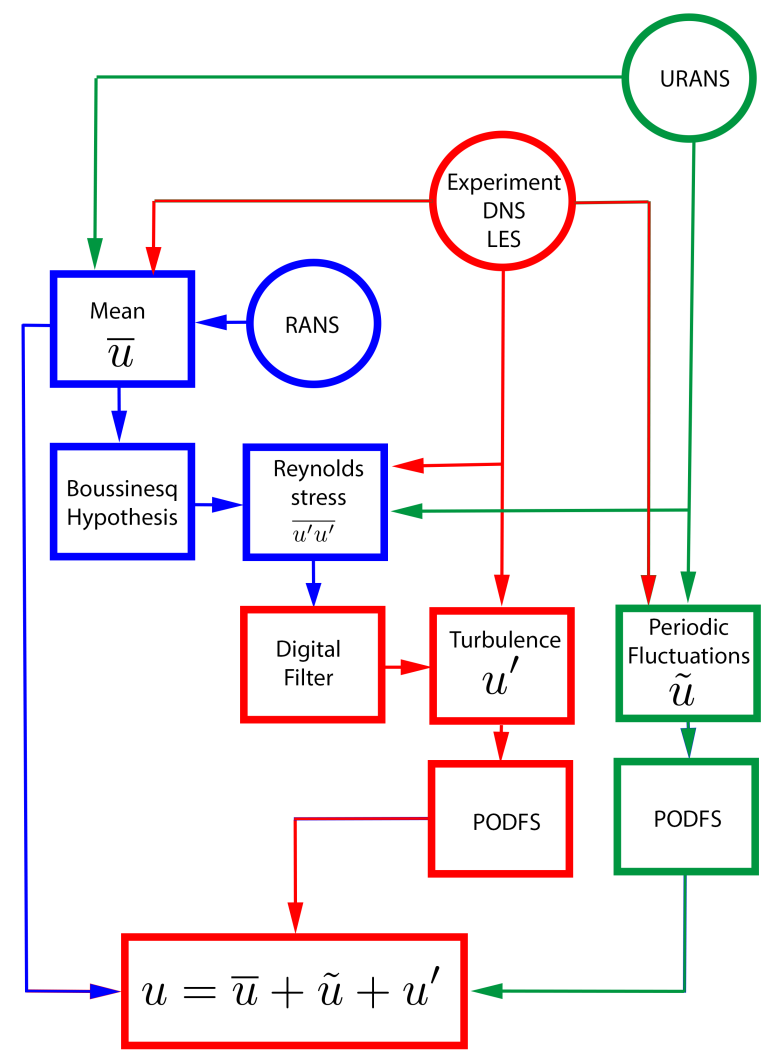

Figure 1: The different ways that experimental and numerical data can be combined with the PODFS and digital filter method to reproduce inlet boundary conditions. Pathways are coloured by relevent contribution. Red: Unsteady/turbulent fluctuations, Green: Periodic/large scale fluctuations and Blue: Temporal mean.

Figure 1 shows how the PODFS method may be integrated with the 
digital filter method and Boussinesq hypothesis to transform mean field data collected from experiment or simulation into a fluctuating turbulent inlet field. Because the PODFS method assumes that the unsteady inlet is a linear combination of modes, multiple PODFS models can be used at once to represent different flow phenomenon. In Section 6, an Unsteady Reynolds averaged Navier-Stokes (URANS) simulation is used to obtain the mean flow field through a jet engine fuel injector. This same simulation was acoustically forced from downstream creating a periodic fluctuation of flow through the injector. This phase locked information was then used to produce a PODFS model for each injector flow passage to represent the acoustic forcing. An incompressible LES simulation was then run on the same geometry using a truncated domain that only included the flow downstream of the injector. In order to account for smaller scale turbulent fluctuations generated in the flow passages, a second PODFS model was developed based on the mean flow field from the same URANS simulation using the Boussinesq hypothesis and the digital filter method and added to the first PODFS model.

Another potential example might be simulating the flow through a single blade row in a gas turbine using LES. Upstream mean field information from a RANS simulation, URANS simulation or experiment could be used to generate both a mean field and turbulent inlet using the digital filter and PODFS method. Alternatively, turbulent information could be provided by a Direct Numerical Simulation (DNS) or LES. Additionally the periodic effects of vane wakes might be captured separately using another PODFS model built using data extracted from a separate URANS simulation or additional experiment. Because the PODFS models are modelled using an analytical function in 
time, each individual donor simulation or experiment that contributes to the inlet data can be run with an independent and therefore optimal timestep with respect to the final simulation.

\section{Channel flow test case}

Following the work of Klein et al. [12], in order to test the method's ability to reproduce the turbulent statistics correctly, the digital filter method was used to generate 100 inlet snapshots based on the DNS channel simulation of Moser et. al. [31] at $R e_{\tau}=395$. The inlet plane had a resolution of $50 \times 500$ cells with the turbulent length scale set to $0.038 h$ where $h$ is the channel height. This corresponds to a turbulent length scale of two cells and a half filter width of four cells. A PODFS reconstruction of the data was also computed using different numbers of modes (out of 100) representing up to $98 \%$ of the fluctuating flow energy to investigate the model sensitivity to mode number. The Fourier energy was set to $E_{b}=0.9$ and then the sensitivity of the solution to this parameter was tested by recomputing the PODFS model with $E_{b}=0.6$. In this case the input profile data $(U, u u, u v, v v, w w)$ was provided as a function of $y / \delta$, the wall normal direction, normalised by the channel half-width. This was completed using the digital_filters.py python program (see Appendix A.

\subsection{Results and discussion}

Figure 2 shows four components of the Reynolds stress tensor for the original DNS channel data, the raw data produced by the digital filter method and the PODFS reconstruction with 41 modes (98\% of the kinetic energy, data compression by a factor of 2) and a PODFS reconstruction with 20 


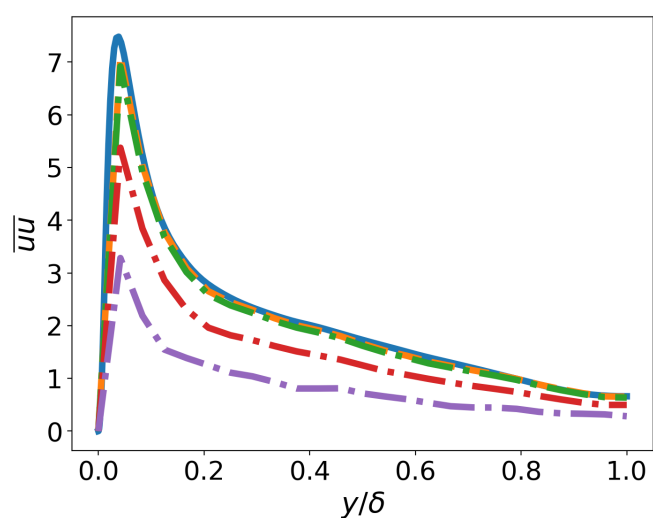

(a)

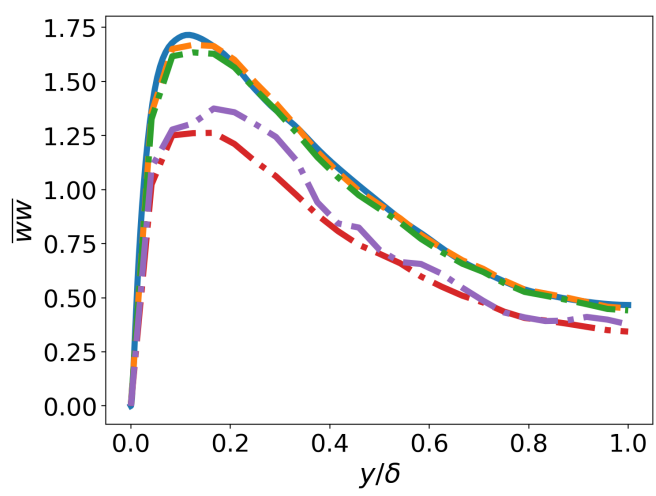

(c)

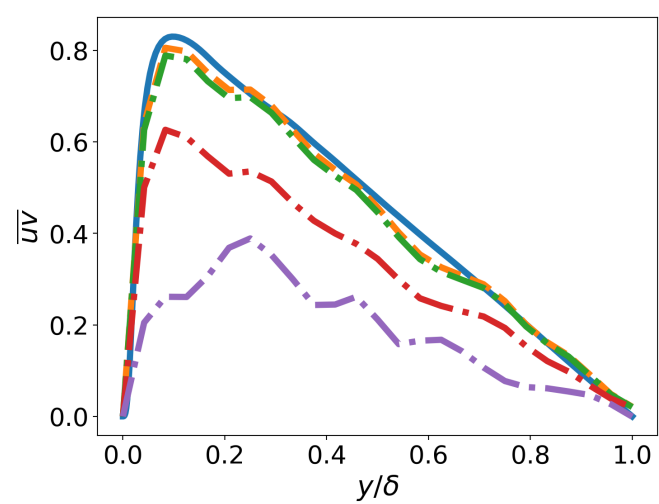

(b)

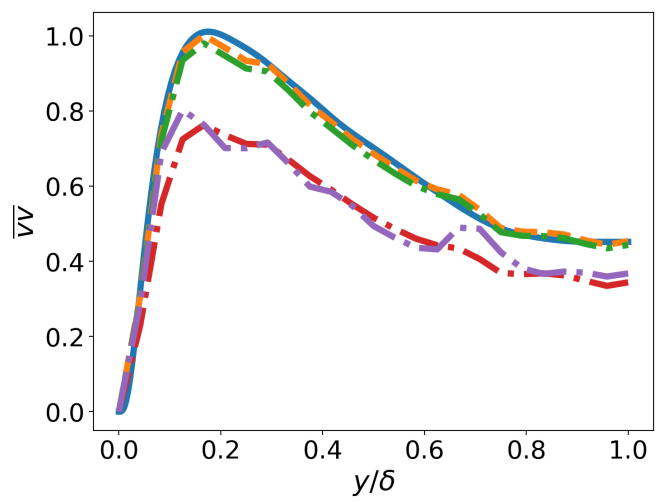

(d)

Figure 2: The original channel flow DNS profile (blue solid line), profile generated using the digital filter method (orange dashed line), the profile generated by the PODFS method with 41 modes and $E_{b}=0.9$ (green chain line), the profile generated by the PODFS method with 20 modes and $E_{b}=0.9$ (red dashed line) and the profile generated by the PODFS method with 41 modes and $E_{b}=0.6$ (purple chain line) for the axial component of Reynolds stress (a), turbulent shear stress (b), span-wise component of Reynolds stress (c) and wall-normal Reynolds stress (d) as a function of the normalised distance from the wall $(y / \delta)$. 
modes (94\% of the kinetic energy, data compression by a factor of 5). This figure shows that the digital filter method closely reproduces the Reynolds stress profile. The PODFS model is seen to match the original digital filter data closely. The mean flow field (not shown) is identical for the four cases. The effect of decreasing the number of POD modes and Fourier coefficients is to reduce the accuracy of the method but with an improved data compression rate.

Figure 3 shows the fluctuating wall-normal velocity at the inlet plane for two instants as generated using the digital filter method and as modelled using the PODFS method (41 modes and $E_{b}=0.9$ ). It can clearly be seen in this case that the large scale structures are closely approximated by the PODFS method.

\section{Turbulent planar jet test case}

The PODFS method was also used to provide a turbulent inlet to a planar jet DNS simulation based on the work of Klein et. al. [12, 13]. The domain is a rectangular prism of dimensions $20 D \times 8 D \times 20 D$ in the axial $(x)$, spanwise $(y)$ and vertical $(z)$ directions where $D$ is the vertical height of the inlet jet which faces the positive axial direction and lies at the midpoint of the domain in the z-direction. To be consistent with [12], the mesh resolution was set to $257 \times 64 \times 512 \approx 8.4 \times 10^{6}$ grid points. The nozzle is resolved using 50 cells and the grid is stretched in the z-direction. The resolution in the other two principle directions is uniform. The Reynolds number was set to $R e=4000$ based on the bulk mean jet velocity $\left(U_{0}\right)$. The inlet jet mean velocity profile was modelled using a hyperbolic-tangent profile such that: 


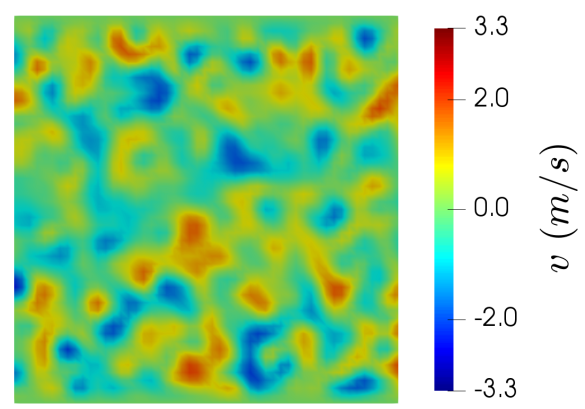

(a)

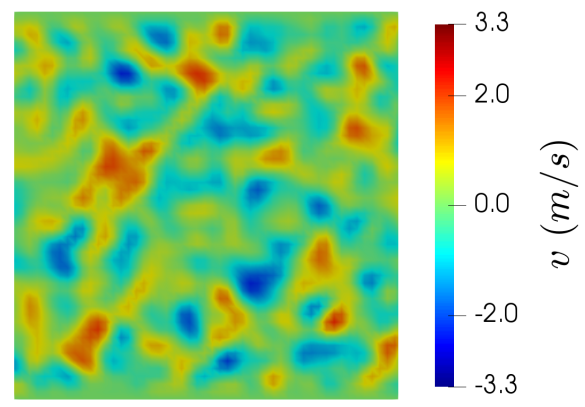

(c)

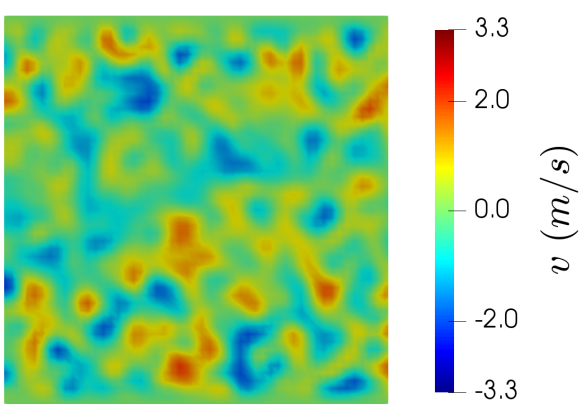

(b)

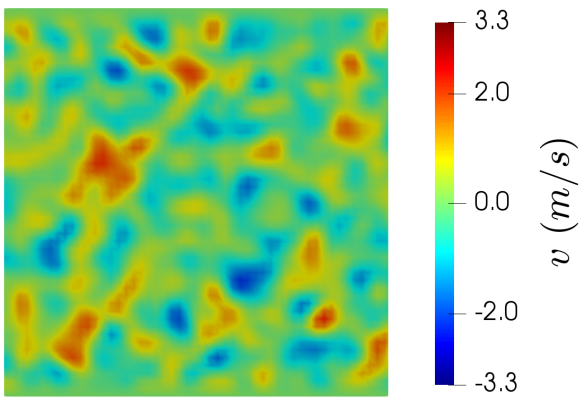

(d)

Figure 3: The wall-normal velocity component over a portion of the inlet at one instant (a), and its PODFS reconstruction (b), the wall-normal velocity component at another instant (c) and its PODFS reconstruction (d). 


$$
\bar{U}=\frac{U_{0}}{2}+\frac{U_{0}}{2} \tanh \left(\frac{z}{2 \theta}\right)
$$

where $\theta$ is the shear layer momentum thickness which was set to $D / \theta=20$. Following Klein [13, the turbulent fluctuation magnitude was assumed to be a simple top-hat profile with $\sqrt{u u} / U_{0}=\sqrt{v v} / U_{0}=\sqrt{w w} / U_{0}=0.02$. 1250 snapshots of the inlet were generated with a turbulent length scale of $1 / 3 D$ in the $\mathrm{y}$ and $\mathrm{z}$ directions and a half filter width of $2 / 3 D$. This choice of filter length and turbulent length scale was also chosen to be consistent with [12]. The time between snapshots was set to be eight times the timestep of the simulation $\left(\delta t_{i n}=8 \delta t\right)$ and the axial turbulent length scale was then scaled by the bulk velocity according to Taylor's hypothesis.

This already highlights an advantage of the PODFS method as it automatically and smoothly interpolates between generated snapshots allowing for flexibility of timestep selection. Generation of the inlet data using the digital filter method at every timestep such that the turbulent length scales were uniform in each principle direction would require the half-filter width to be eight times longer making the method extremely expensive. This cost can be reduced by generating the snapshots with an assumed timestep much larger than the simulation and using an interpolation strategy, with a penalty for accuracy. If the snapshots are generated with too large a timestep then the axial filter width and the axial correlation of the fields breaks down (see the end of Section 2.2. The timestep should therefore be chosen such that the filter size is the same order of magnitude in the three principle directions.

The PODFS reconstruction was achieved using 50 modes representing $97 \%$ of the fluctuating flow energy with the Fourier energy set to $E_{b}=0.9$. 
This represents a reduction of the amount of data that must be transported, saved and loaded in the simulation by a factor of almost 25 . This reduction is tempered by the inclusion of a small control file which communicates the number of modes, timestep and Fourier coefficients for each mode which must be loaded once at the beginning of the simulation. This reduction of data is very effective at speeding up simulations where new inlet data must be applied at each timestep. In [25] the PODFS method was responsible for a speed-up of 2.7 times as compared to the case where individual files are loaded for each inlet at each timestep as is done in the strong recycling method [25].

\subsection{Numerical methodology}

The simulation was completed using the incompressible version of the PRECISE-UNS code [32, an unstructured, finite volume CFD code developed by Rolls-Royce. Spatial differencing was achieved using a second order linear upwind method with an Van Albada limiter [33] for the velocity variables and a symmetrical second order method for pressure. Temporal integration was achieved using a second order backwards method. The timestep was set such that the CFL number $(\Delta t U / \Delta x)$ was less than $1 / 20$ [34].

The PODFS method was used to apply the fluctuating velocity fields to the inlet while the regions of the same boundary above and below the inlet were set to be slip wall boundary conditions. The boundaries at each extreme in the spanwise direction were set to be periodic while all the other boundaries were set to be a convective outflow condition. This is different to the cases of Klein et al. [13, 12] where a Neumann boundary condition was applied at the outlet and a constant pressure condition at the top and bottom boundaries. 
The CFD code used here has only a constant pressure Neumann boundary condition which was found to introduce unwanted vorticity into the field from the top, bottom and outlet boundaries. The use of a convective boundary condition prevents mass entrainment which leads to a slight recirculation of the flow in the domain.

In order to highlight the advantages of the PODFS and digital filter method over the pure digital filter method, the simulation was first run for a total of $9 t^{*}$, using the PODFS digital filter method to provide the initial conditions, before being run a further $8 t^{*}$ where flow statistics were computed. The simulation was then returned to the state it was in at $t=9 t^{*}$ and turbulence was applied to the inlets using the original data from the digital filter that was used to build the PODFS model for another $8 t^{*}$. In this test case $t^{*}$ is the flow through time based on the mean axial velocity at the inlet and the axial length of the domain. Because the PODFS model was built from digital filter data computed at intervals of $8 \times \Delta t$ and in order to have fields for every timestep, the temporal resolution was increased by 8 times using linear interpolation.

\subsection{Results and discussion}

Figure 4 shows the growth of velocity fluctuations at the centreline of the jet and the degradation of the mean flow velocity at the centreline of the jet $\left(U_{c l}\right)$ as it progresses downstream. The fluctuation of axial velocity plot shows that from the initially introduced turbulent fluctuations, the centreline axial velocity fluctuations grow linearly from $x / D \approx 1$. It can be seen in [12] that not sufficiently well correlated (random) fluctuations results in the turbulence dissipating very quickly leading to a laminar flow jet that doesn't 


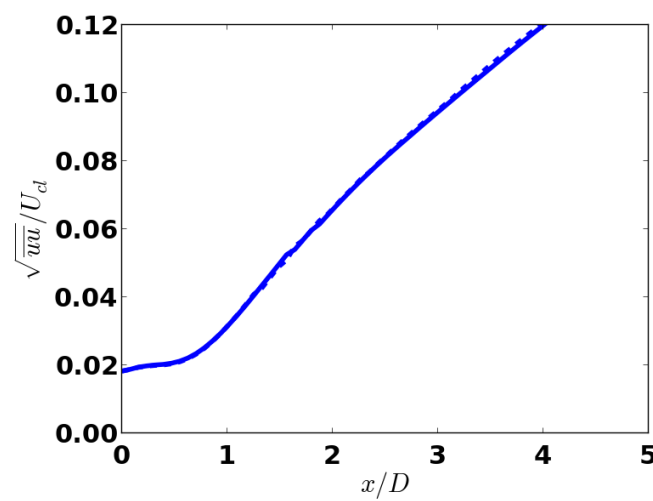

(a)

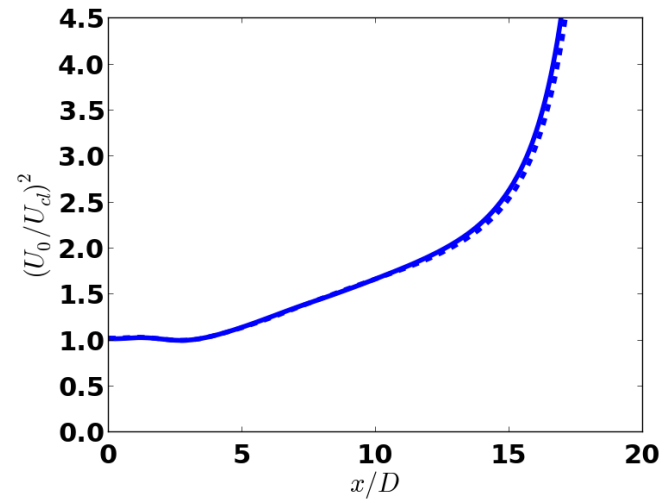

(b)

Figure 4: The centreline axial velocity fluctuations normalised by the centreline mean velocity (a) and the square of the inverse of the centreline mean axial velocity normalised by the initial inlet velocity (b) as a function of the axial location of the jet. The solid line is the PODFS method and the dashed line the original digital filter data.

transition to turbulence before 2-4 jet widths downstream. The results using the original digital filter data is also included which shows that in this case the velocity fluctuations grow at an almost identical rate to the PODFS representation of the same inlet data.

Similarly, the centreline velocity begins to degrade after $x \approx 5 D$. This is consistent with the turbulent jet results in [12] and [13]. In the case of a laminar jet, the centreline velocity does not degrade until $x \approx 8 D$ showing that the PODFS method is generating a turbulent inlet boundary condition. The results using the original digital filter data show that in this case the jet centreline velocity degrades at the same rate as the PODFS case.

The jet is seen to be self-similar between $x \approx 5 D$ and $x \approx 12 D$ before the jet is seen to suddenly flare outwards near the outlet boundary due to 
the recirculation caused by the convective outlet boundary condition.

In the self-similar region the centreline velocity should degrade in the axial direction according to:

$$
\left(\frac{U_{0}}{U_{c l}}\right)^{2}=C_{u}\left(\frac{x}{D}-C_{u, 0}\right)
$$

where $C_{u}$ and $C_{u, 0}$ are constants. Fitting this equation to the simulation data in the region of similarity results in $C_{u} \approx 0.137$ which is lower than the calculation by Klein et. al. [13] (0.178) but higher than the study of Hussain et. al. [35] (0.123).

Similarly, the half width of the jet $\left(z_{1 / 2}\right)$ should evolve downstream as:

$$
\frac{z_{1 / 2}}{D}=C_{z}\left(\frac{x}{D}-C_{z, 0}\right)
$$

Fitting this equation to the data results in $C_{z} \approx 0.136$ which is slightly higher than both the studies of Klein et. al. [13] (0.106) and Hussain et. at. [35] (0.118). This discrepancy may be due to the recirculation of the flow inside the domain. This study also uses a less refined mesh with respect to [13].

Figure 5 shows two components of mean velocity and two components of RMS of velocity across the width of the jet at several axial locations in the self similar region. The width of the jet has been normalised by the jet half-width. The results are also compared to the DNS results of Stanley et. al. [28] which are designated as $D N S, S S M$ in the figures. The mean axial velocity profiles show reasonable agreement with the Stanley et. al. results. The agreement appears to improve as the jet evolves downstream which suggests that if the domain was extended or the outlet boundary condition improved, then the 


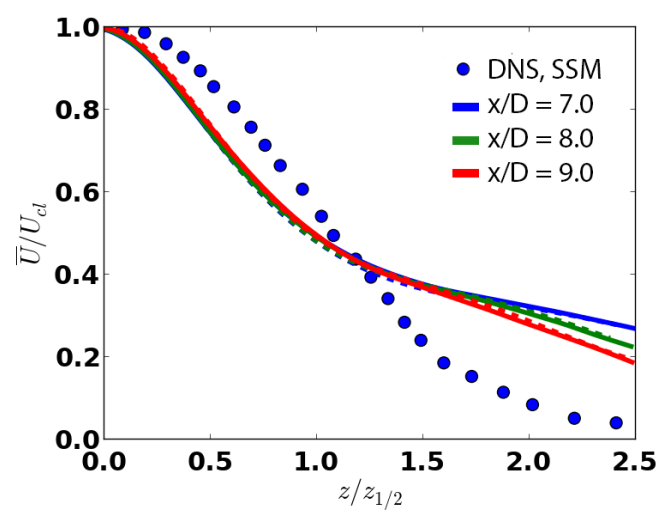

(a)

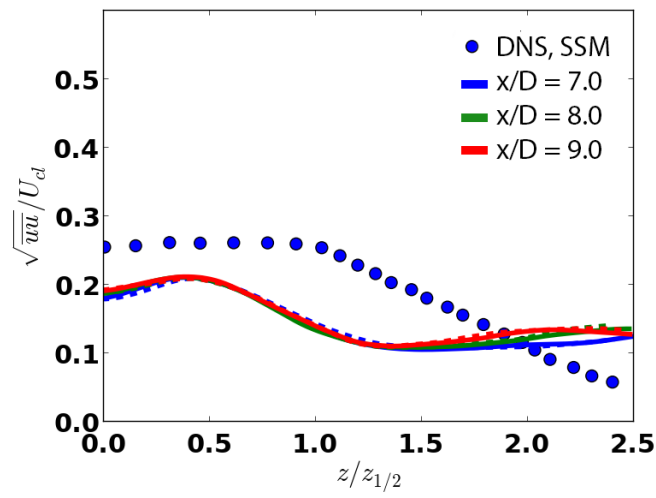

(c)

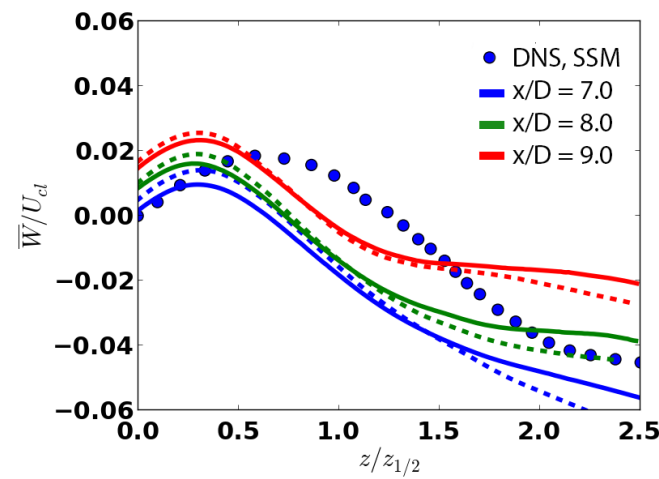

(b)

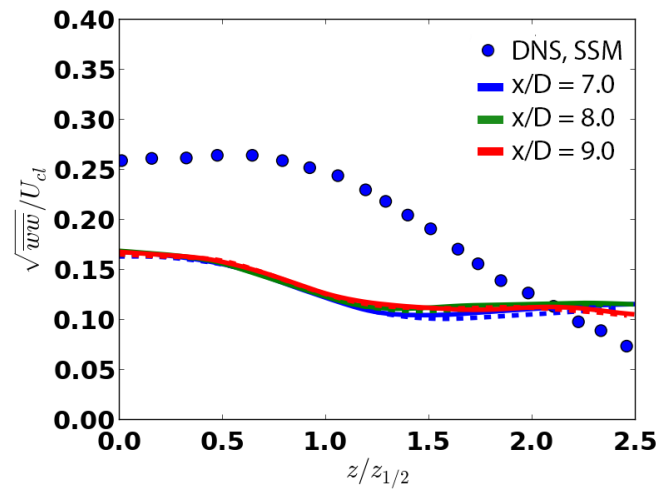

(d)

Figure 5: The mean axial velocity (a), mean lateral velocity (b), axial velocity fluctuations (c) and lateral velocity fluctuations (d) as a function of normalised lateral coordinate across the jet for various axial locations. The original digital filter data is the dashed lines while the solid lines use the PODFS method. 
result would be better. The profile of mean velocity in the lateral direction shows that the jet is spreading out more quickly than the SSM case which may be due to the recirculation zone formed by the choice of boundary condition. The plot of $\sqrt{\overline{u u}}$ shows quite good agreement with the SSM case in the core of the jet but the fluctuations at the edge of the jet are higher than expected. Again this is probably caused by fluctuations at the exit that recirculate back to the edge of the jet which enhances the mixing out of the jet at the jet boundaries. The prediction of $\sqrt{\overline{w w}}$ also appears to be lower than the case of Stanley et. al. [28] which is consistent with what was observed by Klein et. al. [13]. It is suggested by Klein et. al. that this is due to a higher initial centreline fluctuation magnitude and also point out that in the case of SSM a weak co-flow was added.

The mean velocity profile in the similarity region closely represents a Gaussian function:

$$
\frac{U(z)}{U_{c l}}=\exp \left[-C\left(\frac{z}{z_{1 / 2}}\right)^{2}\right]
$$

where $C$ is a constant. Fitting this profile to the data at $x=10 D$ results in $C \approx 0.77$ which again is slightly higher than the value found by Klein et. al. [13] (0.683). Referring again to the shape of the mean profiles, it is clear that the velocity at the edge of the jet is higher than expected thanks to the recirculation zone which is formed by the choice of outlet boundary condition.

The simulation using the original digital filter data also shows similar results to the simulation using the PODFS inlet. The only significant deviation between the two simulations is the lateral mean velocity on the edges of the 
jet, again likely caused by the unstable recirculation zone. The simulation using the pure digital filter data took 0.64 CPUh per timestep as opposed to the PODFS method which achieved 0.26 CPUh per timestep. The PODFS method was in this case 2.5 times faster, this speed-up is due to the time it takes to load the individual inlet files which is much slower than computing the PODFS reconstruction of the velocity fields at each time step. During the loading of the turbulent inlet, all of the processors that do not manage part of the inlet domain must wait for those processes that do manage part of the inlet domain to load and interpolate the turbulent inlet data.. The storage size for the PODFS fields was $90.2 \mathrm{MB}$ as compared to the $2250 \mathrm{MB}$ required by the pure digital filter method before the temporal interpolation was applied. Although only $2250 \mathrm{MB}$ of data had to be saved for the simulation, after interpolation, $18000 \mathrm{MB}$ of data had to be loaded during the simulation. These results are highlighted in Table 1 .

Table 1: Approximate cost of each simulation method in CPUh and the amount of memory that must be loaded during a 10,000 step simulation of the planar jet.

\begin{tabular}{lll}
\hline Method & Memory Loaded (MB) & CPUh \\
\hline Digital filter & 18000 & $6.4 \mathrm{k}$ \\
Digital filter \& PODFS & 90.2 & $2.6 \mathrm{k}$ \\
\hline
\end{tabular}

\section{Acoustically forced fuel injector flow test case}

In order to show one practical application of the method, an acoustically forced swirl stabilised injector flow is modelled using the PODFS method. 
The main motivation for this method is related to obtaining the Flame Transfer Function (FTF) for such a fuel injector. In order to obtain the FTF, the injector must be acoustically forced and the heat release rate and injector mass flow rate be recorded over a length of time. Simulations aimed at obtaining the FTF must therefore be able to model acoustic effects and combustion accurately [36]. The state of the art method for doing this is through the use of compressible reacting LES which caries a significant cost. In such an LES, the injector, upstream and downstream plenums must be meshed sufficiently finely for the acoustic waves and the flame to be resolved accurately.

Compressible simulation domain

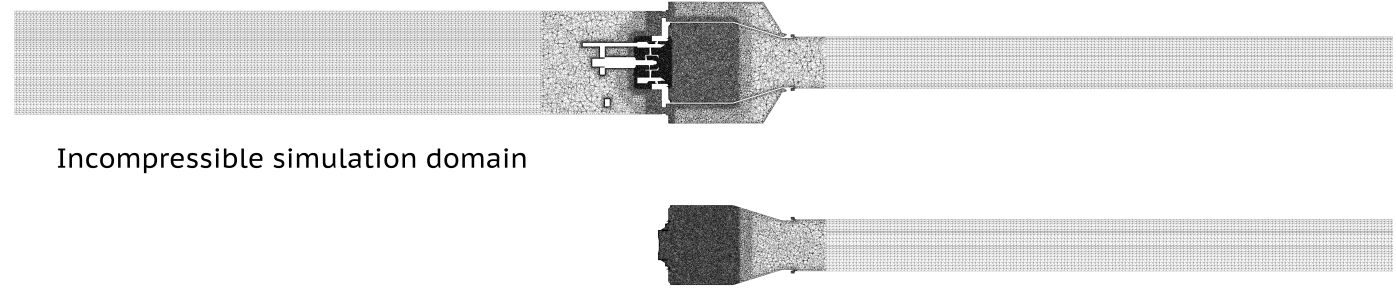

Figure 6: An example of by how much smaller an incompressible simulation domain can be as compared to a compressible domain.

By using an incompressible LES method, the upstream plenum and injector swirl passages can be removed from the computational domain and the mesh in the downstream plenum coarsened which significantly reduces the number of cells (see Figure 6). The lack of a compressible method also accelerates the convergence of a solution as acoustic waves, which must ordinarily propegate from one side of the domain to the other, are not present in the incompressible case. An example of by how much faster an incompressible method might be to a compressible method is provided in Table 2. 
It shows that a compressible reacting simulation of the entire domain would require approximately 8.5 times more CPUh than the equivalent incompressible simulation. The ability of the incompressible simulation to reproduce the compressible simulation results depends crucially on how well the inlet boundary conditions capture the flow coming out of the fuel injector. In [25] it was shown that the PODFS method can be used to capture the acoustic fluctuations through the injector and here it is demonstrated that the use of the PODFS and digital filter method to reproduce the inlet turbulent fluctuations also successfully captures the downstream mean field.

Table 2: Approximate cost of each simulation method in CPUh.

\begin{tabular}{llll} 
Method & Cells & Timesteps & CPUh \\
\hline Reacting compressible LES & $15 \mathrm{M}$ & $100 \mathrm{k}$ & $146 \mathrm{k}$ \\
Reacting incompressible LES & $6 \mathrm{M}$ & $40 \mathrm{k}$ & $17 \mathrm{k}$ \\
\hline
\end{tabular}

The geometry of the fuel injector is described in [25, 37]. It is a swirlstabilised lean-burn fuel injector for use in large civil jet engines. It comprises of three air flow and swirl passages, concentrically located inside the round injector body. In [25] it was shown that the PODFS method could, using incompressible URANS simulations, reproduce the acoustically excited flow field produced by using a compressible URANS method detailed in [37]. However in [38, the application of the mean field to the inlets of an LES simulation resulted in the incorrect penetration of the central jet (see Figure 7). It has been observed in [39] that in a similar fuel injector case, the penetration of the central jet is driven by the relative mass flow rates between the injector flow passages and the level of turbulence in the jets. In this paper it 
is demonstrated that in the isothermal case, the addition of turbulence using the PODFS and digital filter method corrects the penetration length of the central jet.
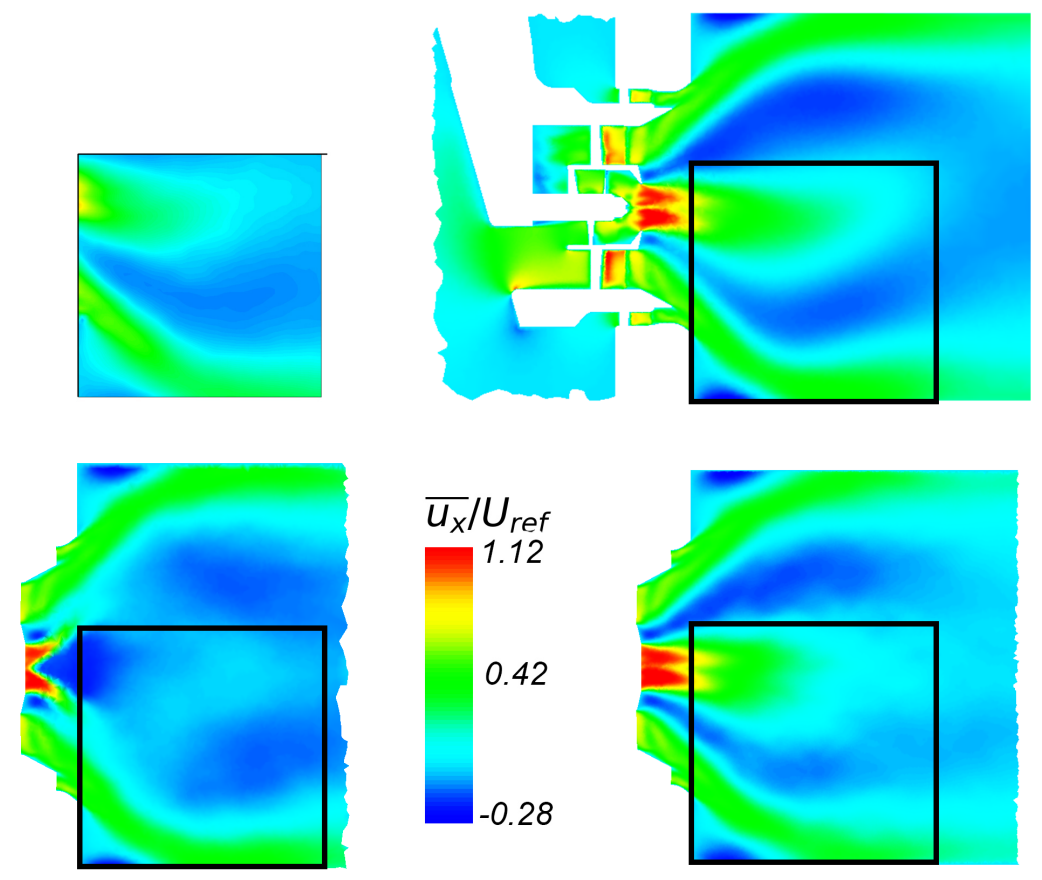

Figure 7: The mean axial velocity from PIV (top left), from the original acoustically forced URANS equation (top right),reproduced from [25], from the PODFS LES calculation built from URANS data only (bottom left) and from the PODFS LES calculation using both URANS data and additional turbulent modes produced using the digital filter (bottom right).

\subsection{PIV}

Isothermal PIV measurements of the flow behind the injector were conducted using a vertically oriented water flow rig at Loughborough University 
[40. The rig consists of a large header tank connected to a long upstream duct that sits above the test section. The distance between the top of the water tank and the bottom of the test section is just over $68 \mathrm{D}$. The injector is mounted to a round orifice that emulates the upstream section of the combustion chamber which sits $55 D$ below the water level of the header tank. Downstream of the injector is the perspex test section with an internal diameter of 1.91D. This is surrounded by a square water jacket to minimise optical refraction with cameras viewing perpendicular to the windows. $1.91 \mathrm{D}$ downstream of the injector is a circular internal blockage that helps to terminate the central recirculation zone and provides a window for imaging the $r \theta$ plane. The rig is shown in Figure 8 . The flow rate of water was maintained at a rate that resulted in a Reynolds number of around one third of that found in the LES simulation but is sufficiently high enough that the flow becomes Reynolds number independent [41].

The PIV light sheet was introduced using two compact Nd/YLF lasers fitted with an articulated mirror arm. The lasers have a maximum energy per pulse of $45 \mathrm{~mJ}$ at a wavelength of $527 \mathrm{~nm}$ and a pulse length of less than $200 \mathrm{~ns}$. The data was captured at a frequency of $\mathrm{St}=54.5$ as this was the maximum repetition rate of the laser system. The laser optics were adjusted to form a light sheet $0.014 D$ thick. 3600 image pairs were captured using a 1 mega-pixel digital camera. The PIV cross-correlation was performed using La Vision DaVis PIV software with a final pass resolution of $32 \times 32$ pixels and $50 \%$ overlap. This resulted in a vector resolution of $0.014 D$. For readers with interest in further details of the experimental methodology please see [42]. References [40], [41] and [42] describe the facility and methodology used in 
this work in significant detail, but as applied to a different fuel injector. Here results are presented by introducing an industrially representative lean-burn injector to that facility which have not been previously reported.

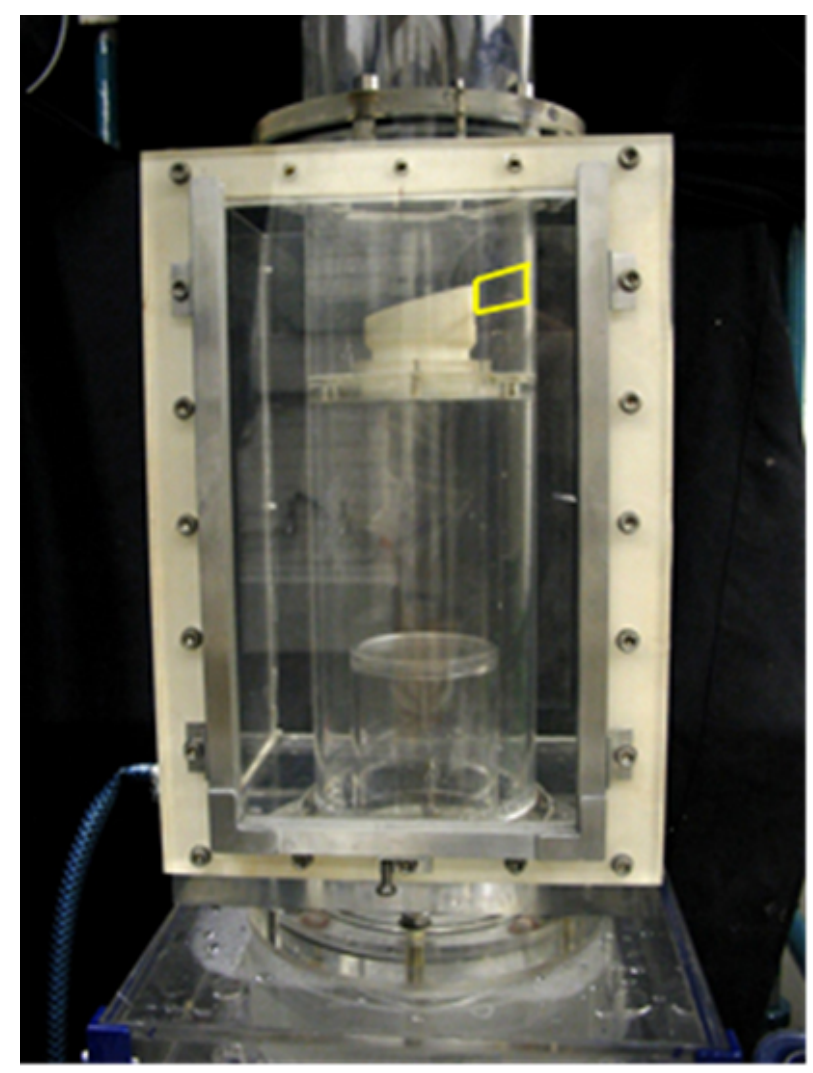

Figure 8: The PIV experimental set-up.

\subsection{Numerical methodology}

The simulation was also completed using the incompressible version of the PRECISE-UNS code [32]. Spatial differencing was achieved using a second order linear upwind method with a Van Albada limiter [33] for the velocity variables and a symmetrical second order method for pressure. The 
turbulence closure was provided by the constant Smagorinsky model [43]. Temporal integration was achieved using a second order backward method. The computational domain is the same as in [25] while the mesh is generated using the same method as in [38] and is shown in Figure 9. The simulations were first run for 4000 steps assuming steady flow and using the $k-\omega$ SST turbulence model before being run as an LES for 65,000 time steps with a timestep of $1 \times 10^{-3} t^{*}$ where $t^{*}=D / U_{\text {ref }}$ and $U_{\text {ref }}$ is a reference velocity. The flow statistics were collected over the last 40,000 steps.

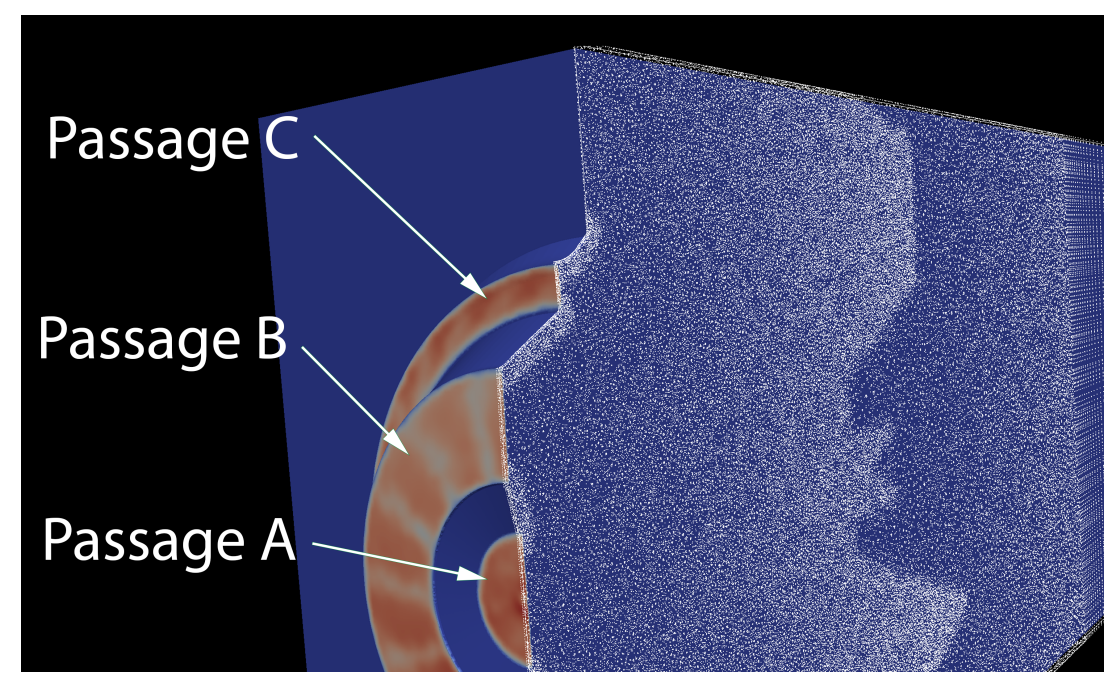

Figure 9: The mesh used in the LES simulation of the fuel injector showing the three inlets where the PODFS models are used to provide the fluctuating inlet flow.

\subsection{Inlet boundary conditions}

There are three inlets (passage A, B and C) into the computational domain and so three different PODFS models were built, one for each inlet. When the flow is computed assuming steady flow, the mean velocity profiles 
extracted from the compressible URANS calculation from [37] are applied to the inlets of the new computation at the exits of the injector flow passages using a linear interpolation strategy. When the flow is allowed to become unsteady, the velocity profiles must be updated at each timestep to represent the changes caused by the acoustic forcing. This was performed using the PODFS method which has been shown to accurately reproduce the mass and swirl number fluctuations in an incompressible URANS simulation [25].

The original compressible URANS simulations from [37] were forced using a $S t=0.3$ acoustic wave run with a timestep of $1 \times 10^{-3} t^{*}$ and at every 20 th time step the velocity fields at the planes of interest were linearly interpolated onto 200x200 point planes, extracted and saved. The PODFS was then built from the 20 most energetic POD modes.

In order to correct the centre jet penetration length (see figure 7), an additional 30 PODFS modes were added, as generated using the digital filter method. In this case the parameters for the digital filter method were generated from the mean velocity profiles. The Reynolds stress profiles were estimated from the mean velocity, turbulent kinetic energy $(k)$ and specific rate of dissipation $(\omega)$ using the Boussinesq eddy viscosity approach (see Equation 9).

A PODFS was calculated from the 30 most energetic modes from 1000 turbulent fields generated using the digital filter method. These 30 PODFS modes were then added to the original 20 PODFS modes that represent the acoustic forcing for each inlet resulting in a 50 mode PODFS model for each inlet. Figure 1 shows how turbulent fluctuations can be represented using one PODFS model which is then added to a second PODFS model which 
represents periodic fluctuations.

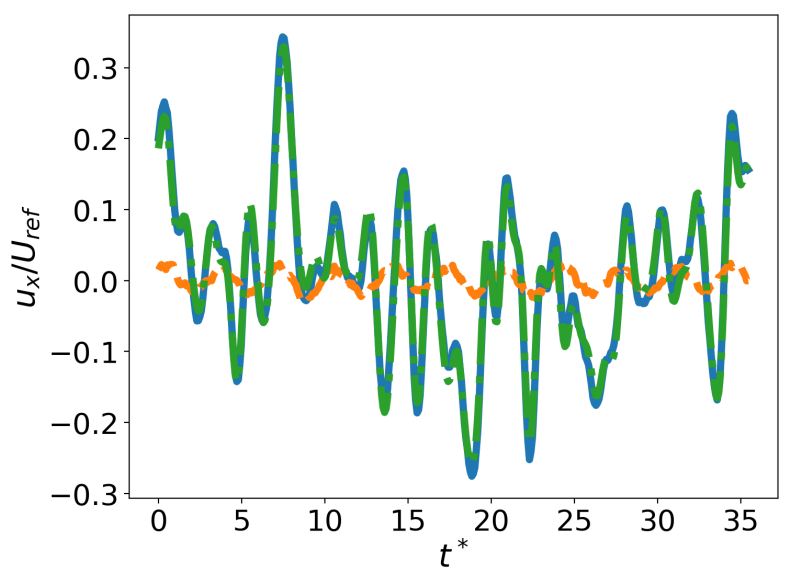

Figure 10: The area averaged axial velocity fluctuations as a function of time at the exit of Passage B using the first 20 PODFS modes which represent the acoustically induced fluctuations of velocity (orange dashed line), the axial velocity fluctuations at a point in the exit plane of Passage B using the last 30 PODFS modes which represent the turbulent fluctuations (green chain line) and the sum of these two contributions (blue solid line).

\subsection{Results and discussion}

Figure 10 shows the relative contributions to the overall velocity fluctuations at the exit of Passage B (one of the three inlets in the incompressible simulation) of the acoustic forcing and the imposed turbulent field. The orange dashed line shows the area averaged velocity fluctuation across the inlet which is represented using the first 20 PODFS modes which come from the compressible forced URANS simulation. The green chain line shows the imposed velocity fluctuations at a point on the inlet plane from the last 30 PODFS modes which are generated using the digital filter technique. The turbulent fluctuations at the inlet are significantly larger in magnitude than 
the velocity fluctuations induced by the acoustic forcing which highlights the importance of including the turbulent fluctuations in any representation of the inlet flow.

For the fuel injector case, Figure 7 shows the mean axial velocity in the combustion chamber for four different cases. The first case is the PIV experiment detailed in Section 6.1. The second is the results of the fully compressible forced URANS simulations described in [37] and presented in 25]. The final two cases are the same LES simulation run with two different PODFS inlets. In the first case the PODFS is built using only the information coming from the compressible URANS simulation without the digital filter. In this case the small scale turbulent structures are not present in the central jet which results in the incorrect jet penetration, the central jet reverts to the flipped configuration seen in [38]. In the final case, the original 20 mode PODFS is supplemented with 30 turbulent modes generated using the digital filter method for each inlet. In this case the central jet penetration is correctly captured and the velocity field is consistent with the fields observed in the PIV and the compressible URANS simulation.

\section{Conclusion}

The PODFS method has been successfully applied to three sets of turbulent inflow data generated by the digital filter method. In the first case the PODFS and digital filter methods were used to successfully represent the channel flow turbulent statistics given by the DNS calculation of Moser et. al. [31]. The application of the PODFS compressed the data by a factor of two. 
The PODFS was then used to approximate the turbulent inflow generated by the digital filter method for a turbulent planar jet simulation. The PODFS compressed the data by a factor of 25 in this case. The turbulent planar jet simulations were run to emulate the results of Klein et. al. [12, 13]. The application of the PODFS and digital filter fields successfully prevents the jet from re-laminarising as it moves downstream and instead the velocity fluctuations are seen to grow after $x / D \approx 1$ and the mean centreline velocity is seen to decay in a self-similar manner after $x / D \approx 5$.

The velocity profile across the jet in the self-similar region is compared against the results found by Stanley et al. 228] which shows reasonable agreement except for the case of $\sqrt{\overline{w w}}$ fluctuations which are under-predicted but consistent with the results of Klein et. al. [13]. The same simulation was run with the original digital filter data before application of the PODFS method and shown to produce almost identical results showing that the PODFS successfully compresses the data and reduces the running time of the simulation without compromising the accuracy.

Finally, in a practical example of how the PODFS and digital filter method may be used in an industrial setting, two PODFS models are combined to reproduce both periodic fluctuations caused by acoustic forcing of the injector and the inflow turbulence. The periodic PODFS model was generated using data from a compressible URANS computation while the turbulent input data was generated using the digital filter method where the Reynolds stress tensor was built from the same compressible URANS simulation mean inlet flow field by using the Boussinesq eddy viscosity approximation. By combining both these PODFS models, the passage $\mathrm{C}$ inlet 
flow was corrected from a 'flipped' to the 'penetrating' configuration seen in experiments and in the URANS simulation.

The PODFS method is shown to be a useful companion to the digital filter method that significantly increases the usefulness and decreases the computational requirements of adding realistic turbulence to the inlets of scale resolved simulations. Furthermore the PODFS can also be used to simultaneously add periodically fluctuating effects to the inlets such as those caused by acoustic waves or the shedding of wakes.

\section{Acknowledgements}

The authors wish to acknowledge the ongoing support of Marco Zedda from Rolls-Royce. This work is financially supported by Rolls-Royce and the EPSRC (Engineering and Physical Sciences Research Council) through the Centre for Doctoral Training in Gas Turbine Aerodynamics (grant ref. EP/L015943/1) and EPSRC grant ref EP/M023893/1.

\section{Appendix A. Use of the digital_filters.py python program}

The python program may be downloaded from:

https://bitbucket.org/digital_filters_podfs/digital_filters_podfs/

and it is called using:

python digitalfilters.py [options] 
Failure to select any options will activate the help menu which is also accessible using the $-h$ or --help options tags. As an example, the case run in Section 4 was run using:

python digitalfilters.py -i re395rij.inp -t $0.005-k 50$-j 500 -l 2 -v -n $100-m$ 41 - e 0.9

where -i re395rij.inp tells the code to load the re395rij.inp input file which contains a table where the columns represent the $y, U, u u, v v, w w, u v$ values to be applied to the inlet, $-t 0.005$ gives the time step, $-k 50$ is the number of cells in the wall normal direction, $-j 50$ is the number of cells in the spanwise direction, $-l \mathbb{2}$ is the turbulent lengthscale, $-v$ is a verbose flag to also output the individual time step data used in the PODFS compression, - $n 100$ is the number of time steps to generate, $-m 41$ is the number of POD modes to save and -e 0.9 is the desired value of $E_{b}$. Further documentation for the code is available at:

https://digital-filters-podfs.readthedocs.io/en/latest/

\section{References}

[1] J. Schlüter, X. Wu, H. Pitsch, S. Kim, J. Alonso, Integrated simulations of a compressor/combustor assembly of a gas turbine engine, in: ASME Turbo Expo 2005: Power for Land, Sea, and Air, American Society of Mechanical Engineers, 2005, pp. 971-982.

[2] A. D. Walker, J. F. Carrotte, J. J. McGuirk, Compres- 
sor/diffuser/combustor aerodynamic interactions in lean module combustors, Journal of Engineering for Gas Turbines and Power 130 (1) (2008) 011504.

[3] J. Li, G. Page, J. McGuirk, Coupled reynolds-averaged navierstokes/large-eddy simulation for outlet guide vane and prediffuser flows, AIAA Journal 53 (3) (2014) 678-691.

[4] J. C. Tyacke, P. G. Tucker, Future use of large eddy simulation in aeroengines, Journal of Turbomachinery 137 (8) (2015) 081005.

[5] X. Wu, Inflow turbulence generation methods, Annual Review of Fluid Mechanics 49 (2017) 23-49.

[6] S. Lee, S. K. Lele, P. Moin, Direct numerical simulation of isotropic turbulence interacting with a weak shock wave, Journal of Fluid Mechanics 251 (1993) 533-562.

[7] X. Wu, J. Schlüter, P. Moin, H. Pitsch, G. Iaccarino, F. Ham, Computational study on the internal layer in a diffuser, Journal of Fluid Mechanics 550 (2006) 391-412.

[8] C. D. Pierce, P. Moin, Progress-variable approach for large-eddy simulation of non-premixed turbulent combustion, Journal of fluid Mechanics 504 (2004) 73-97.

[9] X. Wu, K. D. Squires, T. S. Lund, Large eddy simulation of a spatiallydeveloping boundary layer, in: Proceedings of the 1995 ACM/IEEE conference on Supercomputing, 1995, pp. 67-es. 
[10] T. S. Lund, X. Wu, K. D. Squires, Generation of turbulent inflow data for spatially-developing boundary layer simulations, Journal of computational physics 140 (2) (1998) 233-258.

[11] W. Bechara, C. Bailly, P. Lafon, S. M. Candel, Stochastic approach to noise modeling for free turbulent flows, AIAA journal 32 (3) (1994) 455-463.

[12] M. Klein, A. Sadiki, J. Janicka, A digital filter based generation of inflow data for spatially developing direct numerical or large eddy simulations, Journal of computational Physics 186 (2) (2003) 652-665.

[13] M. Klein, A. Sadiki, J. Janicka, Investigation of the influence of the reynolds number on a plane jet using direct numerical simulation, International journal of heat and fluid flow 24 (6) (2003) 785-794.

[14] A. Saghafian, L. Shunn, D. A. Philips, F. Ham, Large eddy simulations of the hifire scramjet using a compressible flamelet/progress variable approach, Proceedings of the Combustion Institute 35 (2) (2015) 21632172 .

[15] E. Touber, N. D. Sandham, Large-eddy simulation of low-frequency unsteadiness in a turbulent shock-induced separation bubble, Theoretical and Computational Fluid Dynamics 23 (2) (2009) 79-107.

[16] N. S. Dhamankar, G. A. Blaisdell, A. S. Lyrintzis, An overview of turbulent inflow boundary conditions for large eddy simulations, in: 22nd AIAA computational fluid dynamics conference, 2015, p. 3213. 
[17] R. H. Kraichnan, Diffusion by a random velocity field, The physics of fluids 13 (1) (1970) 22-31.

[18] M. Hoshiya, Simulation of multi-correlated random processes and application to structural vibration problems, in: Proceedings of the Japan Society of Civil Engineers, Vol. 1972, Japan Society of Civil Engineers, 1972, pp. 121-128.

[19] M. Karweit, P. Blanc-Benon, D. Juvé, G. Comte-Bellot, Simulation of the propagation of an acoustic wave through a turbulent velocity field: A study of phase variance, The Journal of the Acoustical Society of America 89 (1) (1991) 52-62.

[20] I. Marušić, A. Perry, A wall-wake model for the turbulence structure of boundary layers. part 2. further experimental support, Journal of Fluid Mechanics 298 (1995) 389-407.

[21] N. Sandham, Y. Yao, A. Lawal, Large-eddy simulation of transonic turbulent flow over a bump, International Journal of Heat and Fluid Flow 24 (4) (2003) 584-595.

[22] N. Jarrin, S. Benhamadouche, D. Laurence, R. Prosser, A syntheticeddy-method for generating inflow conditions for large-eddy simulations, International Journal of Heat and Fluid Flow 27 (4) (2006) 585-593.

[23] P. Subbareddy, D. Peterson, G. Candler, I. Marusic, A synthetic inflow generation method using the attached eddy hypothesis, in: 24th AIAA Applied Aerodynamics Conference, 2006, p. 3672. 
[24] A. Spille-Kohoff, H.-J. Kaltenbach, Generation of turbulent inflow data with a prescribed shear-stress profile, Tech. rep., Technische University Berlin (Germany) Hermann-Fottinger Inst Für Stromungsmechanik (2001).

[25] N. C. W. Treleaven, J. Su, A. Garmory, G. J. Page, An efficient method to reproduce the effects of acoustic forcing on gas turbine fuel injectors in incompressible simulations, Flow, Turbulence and Combustiondoi: 10.1007/s10494-019-00020-4.

URL https://doi .org/10.1007/s10494-019-00020-4

[26] P. Druault, S. Lardeau, J.-P. Bonnet, F. Coiffet, J. Delville, E. Lamballais, J.-F. Largeau, L. Perret, Generation of three-dimensional turbulent inlet conditions for large-eddy simulation, AIAA journal 42 (3) (2004) $447-456$.

[27] L. Perret, J. Delville, R. Manceau, J.-P. Bonnet, Turbulent inflow conditions for large-eddy simulation based on low-order empirical model, Physics of Fluids 20 (7) (2008) 075107.

[28] S. Stanley, S. Sarkar, J. Mellado, A study of the flow-field evolution and mixing in a planar turbulent jet using direct numerical simulation, Journal of Fluid Mechanics 450 (2002) 377-407.

[29] G. K. Batchelor, The theory of homogeneous turbulence, Cambridge university press, 1953.

[30] L. Sirovich, Turbulence and the dynamics of coherent structures. i. co- 
herent structures, Quarterly of applied mathematics 45 (3) (1987) 561571.

[31] R. D. Moser, J. Kim, N. N. Mansour, Direct numerical simulation of turbulent channel flow up to re $\tau=590$, Physics of fluids 11 (4) (1999) 943-945.

[32] M. Anand, R. Eggels, M. Staufer, M. Zedda, J. Zhu, An advanced unstructured-grid finite-volume design system for gas turbine combustion analysis, in: ASME 2013 Gas Turbine India Conference, American Society of Mechanical Engineers, 2013, pp. V001T03A003V001T03A003.

[33] G. Van Albada, B. Van Leer, W. Roberts, A comparative study of computational methods in cosmic gas dynamics, in: Upwind and HighResolution Schemes, Springer, 1997, pp. 95-103.

[34] S. B. Pope, Turbulent flows (2001).

[35] A. Hussain, A. R. Clark, Upstream influence on the near field of a plane turbulent jet, The Physics of Fluids 20 (9) (1977) 1416-1426.

[36] S. Candel, D. Durox, T. Schuller, J.-F. Bourgouin, J. P. Moeck, Dynamics of swirling flames, Annual review of fluid mechanics 46 (2014) $147-173$.

[37] N. C. W. Treleaven, J. Su, A. Garmory, G. J. Page, The response to incident acoustic waves of the flow field produced by a multi-passage lean-burn aero-engine fuel injector, in: ASME 2017 Turbine Technical 
Conference and Exposition, American Society of Mechanical Engineers, 2017, pp. GT2017-64527.

[38] N. C. W. Treleaven, J. Su, A. Garmory, G. J. Page, The effect of sauter mean diameter fluctuations on the heat release rate in a lean-burn aeroengine combustor, in: ASME Turbo Expo 2019: Turbomachinery Technical Conference and Exposition, American Society of Mechanical Engineers, 2019, pp. GT2019-90321.

[39] M. A. Williams, J. F. Carrotte, A. J. Moran, A. D. Walker, Impact of upstream boundary conditions on fuel injector performance in a low trl reacting flow experimental facility, in: ASME Turbo Expo 2018: Turbomachinery Technical Conference and Exposition, American Society of Mechanical Engineers, 2018.

[40] K. Midgley, A. Spencer, J. J. McGuirk, Unsteady flow structures in radial swirler fed fuel injectors, Journal of engineering for gas turbines and power 127 (4) (2005) 755-764.

[41] L. Cheng, A. Spencer, Residence time measurement of an isothermal combustor flow field, Experiments in fluids 52 (3) (2012) 647-661.

[42] A. Spencer, J. J. McGuirk, K. Midgley, Vortex breakdown in swirling fuel injector flows, Journal of Engineering for Gas Turbines and Power 130 (2) (2008) 021503.

[43] J. Smagorinsky, General circulation experiments with the primitive equations: I. the basic experiment, Monthly weather review 91 (3) (1963) 99-164. 